\title{
An Immune Dot-Blot Test for the Diagnosis of Ocular Infection with Chlamydia trachomatis
}

\author{
P. N. BISHOP, A. B. TULLO, R. KILLOUGH, S. J. RICHMOND \\ Manchester
}

\begin{abstract}
Summary
We report a new simple non culture technique for the diagnosis of chlamydial eye disease. The immune dot-blot test (IDBT) detects chlamydial lipopolysaccharide (LPS) antigen which, after being trapped on nitrocellulose membrane, is detected by autoradiography with ${ }^{125}$ I-labelled genus specific monoclonal antibody. This test was evaluated over a two year period in adults and neonates, by comparing it to culture, serological detection of chlamydial antibodies and clinical features. We demonstrate that the IDBT is more than twice as sensitive as culture, and suggest that in order to achieve a reliable diagnosis of chlamydial eye infection an immunological test for chlamydial antigen should be used in preference to tests which detect the organisms themselves.
\end{abstract}

Chlamydia trachomatis is a common cause of sexually transmitted disease which is estimated to account for over $50 \%$ of cases of non-specific urethritis. ${ }^{1}$ This large reservoir of infection may result in ocular disease in sexually active young adults with genito-urinary tract infection, ${ }^{2}$ and in neonates whose mothers are infected. ${ }^{3}$ It is a relatively uncommon cause of kerato-conjunctivitis in adults, ${ }^{4,5,6,7}$ but a common cause of ophthalmia neonatorum. ${ }^{3,4,8}$ Chlamydial infection is an important cause of morbidity in both neonates and adults, particularly if allowed to run a prolonged course. ${ }^{2,8,9}$ It is therefore important to use a sensitive and efficient laboratory test for this condition.

Culture is relatively insensitive for this purpose. Not only can the natural eye infection persist in the absence of recoverable organisms ${ }^{10}$ but topical chloramphenicol treatment, which is often given before swabs for chlamy- dial diagnosis are taken, also makes isolation difficult since it suppresses infectivity without eradicating the organisms. ${ }^{9,11,12}$ Nevertheless under these circumstances chlamydia infected cells probably continue to excrete the chlamydia lipopolysaccharide (LPS) antigen. ${ }^{13} \mathrm{~A}$ sensitive test for this antigen may therefore reveal more infections than those identified by culture alone. We report the results of a two year assessment of a recently developed immune dot-blot test (IDBT). ${ }^{14}$ In this test, which has already been shown to be a sensitive alternative to culture for the diagnosis of genital chlamydial infection, chlamydial LPS is detected with a radiolabelled monoclonal antibody directed against a chlamydia-specific epitope of LPS antigen.

\section{Materials and Methods}

All conjunctival swabs obtained at Manchester Royal Eye Hospital between August

From: The Department of ${ }^{1}$ Ophthalmology, University of Manchester, and the ${ }^{2}$ North Manchester Regional Virus Laboratory, Booth Hall Childrens Hospital, Manchester, England.

Correspondence to: Mr P N Bishop, Department of Ophthalmology, Lister House, Manchester Royal Eye Hospital, Oxford Road, Manchester, M13 9WH. 
1987 and July 1989 from patients suspected of chlamydial eye disease were evaluated by the IDBT and culture. All patients also had routine bacteriology investigations. The conjunctival swabs for chlamydia diagnosis were immediately put in sucrose phosphate transport medium. ${ }^{15}$ The majority of specimens were held at $4^{\circ} \mathrm{C}$ and cultured by a standard technique $^{15}$ within 24 hours. When this was not possible specimens were stored in liquid nitrogen until they were tested. Both culture and the IDBT ${ }^{14}$ were carried out on all specimens. In the latter test specimens are treated with proteinase $\mathrm{K}$, then chlamydial LPS antigen is trapped on nitrocellulose membrane and detected by autoradiography with an ${ }^{125} \mathrm{I}$ labelled monoclonal antibody. Daily analysis of up to 90 specimens is routine, the test effectively taking two working days to complete, largely because the autoradiography stage takes 24 hours.

Adult patients who proved to be positive by either method were, on follow up, investigated serologically for fluorescent antibodies to $C$. trachomatis in a test which uses whole chlamydial inclusions as antigen. ${ }^{15}$ All patients, their consorts and the parents of affected neonates were treated with either systemic tetracycline for adults or erythromycin syrup for neonates, for a period of three weeks.

\section{Results}

Over the two year period, specimens from 621 patients over six months of age and from 91 babies less than six months old were examined. Twenty eight of 621 specimens were positive by IDBT including six that were also positive in culture. These 28 specimens came from patients whose ages ranged from 15 to 43 years (mean 22 years). Therefore, 22 adults were IDBT positive but culture negative; Clinical records were available on 21 of these adults, all of whom had typical clinical features of chlamydial eye disease and were similar to the IDBT and culture positive group (Table I), except that significantly more culture negative patients were on treatment when the conjunctival swabs were taken $(p<0.01$, Fishers exact test). Fourteen of the IDBT positive, culture negative adults were tested serologically and all had significant levels of chlamydial antibodies (Table I).
Thirty two of the 91 specimens from babies under six months of age were positive by IDBT, including 11 that were also positive for culture. The ages of the 32 babies at presentation ranged from six to 42 days (mean 14 days). Therefore specimens from 21 babies were positive by IDBT, but culture negative; The clinical records of 18 of these 21 babies were located, and they were compared with the clinical records of the 11 culture and IDBT positive babies. All had a history and showed clinical signs typical of chlamydial ophthalmia (Table II). As in the affected adults, significantly more culture negative babies were on treatment at the time they were swabbed than the culture positive group $(\mathrm{p}<0.01$, Fishers exact test).

In total, sixty patients were positive by IDBT. Pathogenic bacteria were concurrently grown from the eyes of three of these patients, all of whom were neonates. Staphylococcus aureus was recovered from all these three swabs and one also grew Streptococcus viridans. In addition, C. trachomatis was also cultured from the eye of one of these babies.

\section{Discussion}

In this study all 17 specimens which yielded $C$. trachomatis in culture were also positive by IDBT, but more than twice as many specimens were positive by IDBT alone. Confirmatory direct immunofluorescent staining tests to detect chlamydial elementary bodies ${ }^{16}$ in the centrifuged deposit from these specimens were not possible since the proteinase $\mathrm{K}$ treatment used in the IDBT test destroys the organisms. Nevertheless we feel that these were true positive results. The 21 adults positive by IDBT alone whose records were available all had typical clinical features and those who were serologically tested all had significant levels of chlamydial antibodies (serological positivity implies current or previous chlamydial infection, although this is not necessarily ocular). Likewise the clinical records of the 18 IDBT positive culture negative neonates, when available, were all commensurate with the diagnosis of chlamydial ophthalmia. Moreover, symptoms resolved once appropriate treatment was instituted. Additional laboratory proof that IDBT positive, culture negative specimens represent 
Table I Clinical features of 21 IDBT positive, culture negative adults, compared with the 6 IDBT and culture positive adults

\begin{tabular}{lcc}
\hline & $\begin{array}{c}\text { IDBT }+v e, \\
\text { culture }-v e\end{array}$ & $\begin{array}{c}\text { IDBT and } \\
\text { culture }+v e\end{array}$ \\
\hline Male: female ratio & $11: 10$ & $4: 2$ \\
Age in years & $15-43$ (Ave 22.2) & $16-26($ Ave 21.1$)$ \\
Duration of symptoms in weeks & $1-12$ (Ave 3.6) & $1-7$ (Ave 3.7) \\
Already on treatment when swabbed & $80 \% *$ & $17 \% *$ \\
Clinical Features: & $23 \%$ & $17 \% *$ \\
Bilateral conjunctivitis & $100 \%$ & $100 \% *$ \\
Follicles & $86 \%$ & $33 \% *$ \\
Keratitis & $48 \%$ & $33 \% *$ \\
Pre-auricular lymph node & $23 \%$ & $0 \% *$ \\
Genitourinary symptoms & $100 \%(14$ tested) & $100 \% *(4$ tested) \\
\hline
\end{tabular}

${ }^{*} \mathrm{p}<0.01$, Fisher exact test (see text)

$\dagger$ Serological titres of chlamydial antibodies were all greater than 1/320, apart from one result of 1/160.

true positives has recently been obtained by showing that chlamydial DNA can usually be demonstrated in these specimens by the polymerase chain reaction (personal communication, Dr Meryl Lusher).

False positive results may occur in tests which rely on immunological detection of chlamydial antigen where $S$. aureus is present in the clinical specimen. This is due to staphylococcal protein $\mathrm{A}$, which is also trapped on the nitrocellulose membrane and which binds to some classes of $\mathrm{IgG}$ (including $\mathrm{IgG}_{2}$, the class of immunoglobulin used in the IDBT). This problem is avoided in the IDBT by pretreatment of all specimens with proteinase $\mathrm{K}$ before they are applied to the membrane. This enzyme destroys protein A but has no effect on the chlamydial LPS antigen. ${ }^{14}$ There was no evidence of such false positive reactions in this study; $S$. aureus was only recovered from three IDBT positive babies, who all had typical chlamydial ophthalmia and one case was confirmed by the culture of $C$. trachomatis.

This work highlighted once more the problem caused by prior treatment with antibiotics when diagnosis relies on culture alone. Significantly more IDBT positive, culture negative specimens were from patients on treatment (usually topical chloramphenicol) at the time of presentation than the culture positive group (Tables I and II).

It became clear during this study that, despite good cooperation with the laboratory, positive results sometimes failed to bring about appropriate action. This was particularly true of adults attending the casualty department, who failed to return to appointments, and of babies who had been discharged because of improvement in signs, but who had not received curative treatment. As a direct result of this study several patients from each group were recalled form completion of appropriate treatment and advice to consorts

Table II Clinical features of 18 IDBT positive, culture negative babies, compared with the 11 IDBT and culture positive babies

\begin{tabular}{lcc}
\hline & $\begin{array}{c}\text { IDBT }+v e, \\
\text { culture }-v e\end{array}$ & $\begin{array}{c}\text { IDBT and } \\
\text { culture }+v e\end{array}$ \\
\hline Male: female & $9: 9$ & $4: 7$ \\
Age at presentation in days & $6-21$ (Ave 13.5) & $6-42($ Ave 14.7) \\
Onset of symptoms in days & $0-10$ (Ave 5.8) & $0-8($ Ave 4.7$)$ \\
Already on treatment when swabbed & $88 \% *$ & $36 \% *$ \\
Clinical Features: & & $63 \%$ \\
Bilateral conjunctivitis & $61 \%$ & $18 \%$ \\
Haemorrhagic discharge & $44 \%$ & $63 \%$ \\
\hline
\end{tabular}

${ }^{*} \mathrm{p}<0.01$, Fishers exact test. 
and mothers. We recommend that no baby should be discharged from care until all the requested results have reached the case notes and that all cases of ophthalmia neonatorum should be assumed to be chlamydial until proven otherwise. Furthermore we recommend that a sensitive method that detects chlamydial antigen should be employed for diagnosis, rather than techniques that rely on the presence of chlamydial elementary bodies.

\section{References}

${ }^{1}$ Thompson SE and Washington AE: Epidemiology of sexually transmitted Chlamydia trachomatis infection. Epidemiologic Reviews 1983, 5: 96-123.

${ }^{2}$ Viswalingam ND, Wishart MS, Woodland RM: Adult chlamydial ophthalmia (paratrachoma). $\mathrm{Br}$ Med Bull 1983, 39: 123-7.

${ }^{3}$ Winceslaus J, Goh BT, Dunlop EMC, Mantell J, Woodland RM, Forsey T, Treharne JD: Diagnosis of ophthalmia neonatorum. Br Med J 1987, 295: 1377-9.

${ }^{4}$ Harding SP, Mallinson H, Smith JLS, Clearkin LG: Adult follicular conjunctivitis and neonatal ophthalmia in a Liverpool eye hospital. Eye 1987, 1: 512-21.

${ }^{5}$ Wishart PK, James C, Wishart MS, Darougar S: Prevalence of acute conjunctivitis caused by chlamydia, adenovirus, and herpes simplex virus in an ophthalmic casualty department. Br J Ophthalmol 1984, 68: 653-5.

${ }^{6}$ Potts MJ, Paul ID, Roome APCH, Caul EO: Rapid diagnosis of Chlamydia trachomatis infection in patients attending an ophthalmic casualty department. Br J Ophthalmol 1986, 70: 677-80.

${ }^{7}$ Tullo AB, Richmond SJ, Easty DL: The presentation and incidence of paratrachoma in adults. $J$ Hyg Camb 1981, 87: 63-9.

${ }^{8}$ Hobson D, Rees E, Viswalingam ND: Chlamydial infections in neonates and older children. $\mathrm{Br} \mathrm{Med}$ Bull 1983, 39: 128-32.

${ }^{9}$ Watson PG and Gairdner D: TRIC agent as a cause of neonatal eye sepsis. Br Med J 1968, 3: 527-8.

${ }^{10}$ Schachter J, Moncada J, Dawson CR, Sheppard J, Courtright P, Said ME, Zaki S, Hafez SF, Lorincz A: Non-culture methods for diagnosing chlamydial infection in patients with trachoma: a clue to the pathoginesis of the disease. J Infect Dis 1988, 158: 1347-52.

${ }^{11}$ Hobson D, Stefanidis D, Rees E, Tait IA: Effects of chloramphenicol on Chlamydia trachomatis infection in neonatal conjunctivitis and in McCoy cell cultures. J Hyg Camb 1982, 89: 457-66.

${ }^{12}$ Ridgway GL and Oriel JD. Bacterial infection in the newborn. Br Med J 1980, 280: 1087.

${ }^{13}$ Richmond SJ: Chlamydial group antigen in McCoy cells infected with Chlamydia trachomatis and Chlamydia psittaci. FEMS Microbiol Lett 1980, 8: 47-50.

${ }^{14}$ Mearns G, Richmond SJ, Storey CC: Sensitive immune dot-blot test for diagnosis of Chlamydia trachomatis infection. J Clin Microbiol 1988, 26: 1810-13.

${ }^{15}$ Richmond SJ: Chlamydia trachomatis infection. In Jephcott AE ed. Sexually transmitted diseases. A rational approach to their diagnosis. Public Health Laboratory Service 1987, pp 42-53.

${ }^{16}$ Woodland RM: Laboratory diagnosis of chlamydial and viral ocular infections. Eye 1988, 2: suppl s70-s80. 